\title{
Complications of femoral neck fracture fixation using a sliding hip screw with a side plate device in patients older than 65 years
}

\section{Tatsuya Mitsugi}

Teikyo University Hospital

Hideaki Miyamoto ( $\square$ MiyamotoH@med.teikyo-u.ac.jp )

Teikyo universitiy school of medicine

Masahiro Izumi

Teikyo University Hospital

Kentaro Matsui

Teikyo University Hospital

Keisuke Ishii

Teikyo University Hospital

\section{Takashi Suzuki}

Teikyo University Hospital

\section{Taketo Kurozumi}

Teikyo University Hospital

\section{Gen Sasaki}

Teikyo University Hospital

\section{Hirotaka Kawano}

Teikyo University Hospital

Yoshinobu Watanabe

"Teikyo Heisei Daigaku"

\section{Research article}

Keywords: complications, femoral neck fractures, periprosthetic Fractures

Posted Date: May 29th, 2020

DOI: https://doi.org/10.21203/rs.3.rs-31310/v1

License: (1) (1) This work is licensed under a Creative Commons Attribution 4.0 International License.

Read Full License 


\section{Abstract}

\section{Background}

A majority of femoral neck fractures are indicated for surgical treatment within the scope of applicable guidelines. However, the reported overall complication rate in elderly patients one year after hip fracture fixation was $33.3 \%$. The purpose of this study was to clarify the incidence of complications one year after femoral neck fracture fixation using a sliding hip screw with a side plate device in elder than 65-year-old patients.

Methods

We studied the results of 115 cases of the internal fixation for femoral neck fractures in patients older than 65 years of age using the Twins device (HOYA Technosurgical Corporation, Tokyo, Japan). We could follow up 39 of these 115 cases at one year after fixation and retrospectively investigated complications in these patients. Research outcomes were mortality, surgical site infection (SSI), loss of reduction, aseptic necrosis, late segmental collapse (LSC), nonunion, periprosthetic fracture, reoperation, and recovery of walking ability.

Results

The patients consisted of 13 men and 26 women (mean age 80.5 years, range 65-104 years). Mortality was observed in four cases (14.2\%), SSI in one case (2.5\%), loss of reduction in six cases (15.4\%), aseptic necrosis in seven cases (17.9\%), LSC in three cases (7.6\%), nonunion in eight cases $(20.5 \%)$, periprosthetic fracture in three cases (7.6\%), and reoperation was performed in seven cases $(17.9 \%)$, respectively. In 28 cases, the patients could walk independently before injury; this decreased to 19 cases after injury (67.9\%).

\section{Conclusions}

In our study, the incidence of loss of reduction rate and periprosthetic fracture rate were relatively high. Other complications of the Twins device evaluated were equivalent to those reported previously.

\section{Background}

Recent estimates suggest that the annual incidence of hip fractures worldwide will increase to 2.6 million in 2025 and 4.5 million in 2050 [1]. A majority of these fractures are indicated for surgical treatment within the scope of applicable guidelines. However, the reported overall complication rate in elderly patients one year after hip fracture with all the currently used methods of fixation was 33.3\% [2].

Biomechanically, a sliding hip screw with a side plate device provides better stabilization than multiple cancellous screws for femoral neck fractures [3]. The side plate provides lateral support and prevents 
toggling of the cancellous screws in the femoral neck, resulting in inferior and posterior displacement of the femoral head relative to the femoral shaft [3].

The purpose of this study was to clarify the incidence of complications one year after femoral neck fracture fixation using a sliding hip screw with a side plate device in patients older than 65 years of age and to evaluate the superiority of this device compared with those reported previously.

\section{Methods}

This study was conducted with the approval of the Institutional Review Board of our institution, and all participants provided written informed consent. We studied the clinical results of internal fixation for femoral neck fractures in patients older than 65 years of age using Twins (HOYA Technosurgical Corporation, Tokyo, Japan), a device that has been used in Japan for the treatment of both displaced and nondisplaced femoral neck fractures (Figure 1).

Between 2011 and 2018, we treated 115 cases of femoral neck fractures using Twins. We could follow up 39 cases of these 115 cases at one year after fixation and investigated complications of the patients retrospectively. We examined the preoperative following Xp variables: Singh's index, Garden classification, and Pauwels angle. Additionally, we examined the femoral neck-shaft angle (NSA) on the anteroposterior (AP) view of the hip pre- and post-operatively.

The research outcomes were mortality, surgical site infection (SSI), loss of reduction, aseptic necrosis, late segmental collapse (LSC), nonunion, periprosthetic fracture, reoperation and recovery of walking ability. SSI was defined as the fulfilment of one of the following four criteria: (1) purulent drainage (2) a positive culture result from wound swab (3) diagnosis made by a surgeon, unless culture result is negative. We defined loss of reduction as a displacement of more than 10 degrees of the post-operative NSA than the pre-operative NSA and/or severe telescoping of more than $10 \mathrm{~mm}$ of the sliding screw. We diagnosed aseptic necrosis of the femoral head by magnetic resonance imaging at six months after fracture and LSC as the collapse of the weight-bearing segment of the femoral head by AP hip radiography at a point during the one-year follow up, respectively. Nonunion was diagnosed by computed tomography. Using these measurements, we compared each Xp variable between the "loss of reduction group" and the "stable group". In addition, we investigated differences each Xp variables between "nonunion group" and "union group". The differences between the Xp variables were analyzed by MannWhitney U-test. Values of $P<0.05$ were considered statistically significant.

All patients followed a similar postoperative protocol consisting of patient mobilization out of bed on day one with full unrestricted weight bearing. All patients were contacted at three, six, and 12 months post operation, or until death, and follow-up information was obtained.

\section{Results}


The patients consisted of 13 men and 26 women (mean age 80.5 years, range 65-104 years). The demographic preoperative Xp variables are presented in Table 1. Mortality was observed in four cases (14.2\%), SSI in one case (2.5\%), loss of reduction in six cases (15.4\%), aseptic necrosis in seven cases (17.9\%), LSC in three cases (7.6\%), nonunion in eight cases $(20.5 \%)$, periprosthetic fracture in three cases (7.6\%), and reoperation was performed in seven cases (17.9\%), respectively. The number of patients who could walk independently before injury was 28 ; this decreased to 19 after injury (67.9\%).

There were no differences in each Xp variable between the "loss of reduction group" and the "stable group"; Singh's index $(P=0.24)$, Garden classification $(P=0.078)$, Pauwels angle $(P=0.56)$, and preoperative NSA $(P=0.26)$. Additionally, there were no differences in each Xp variable between the "nonunion group" and the "union group"; Singh's index $(P=0.37)$, Garden classification $(P=0.11)$, Pauwels angle $(P=0.57)$, and preoperative NSA $(P=0.14)$.

\section{Discussion}

Previously reported studies showed that complication rates one year after osteosynthesis for femoral neck fracture in elderly patients were as follows: mortality (12.7\%) [4], SSI (1\%) [5], loss of reduction (4.3\%) [5], aseptic necrosis (4-39\%) [6, 7], LSC (12.5\%) [8], nonunion (8.9\%) [9], periprosthetic fracture (1$2 \%$ ) [5]. In our study, the incidence of loss of reduction rate and periprosthetic fracture rate were relatively high. Our definition of "loss of reduction" included telescoping of more than $10 \mathrm{~mm}$ because Slobogean et. al. showed that more than $10 \mathrm{~mm}$ telescoping of the hip screw resulted in disfunction of gluteus muscle power, which can lead to the inability to walk [10]. In our study, although three of the six "loss of reduction" cases could walk before fracture, two of them were unable to walk for a year. The definition of "Ioss of reduction" differs across the reported studies. Therefore, it is difficult to compare the loss of reduction rate to those reported in other studies.

In contrast, other complication rates were almost equivalent to those reported previously $[4,6,7,8,9]$. Four cases of mortality were due to deteriorating pre-existing disease. From our results, none of the Xp variables (Singh index, Garden classification, and Pauwels angle) were responsible for the incidence of loss of reduction or bone union. These complications may have instead been caused by reoperation. Details of the cases of reoperation are shown in Table 2.

Six of the eight patients with nonunion were reoperated by bipolar head replacement or total hip arthroplasty. The other two patients, who were wheelchair-bound, recovered to the prefixation physical status conservatively within six months after primary fixation. All the three periprosthetic fracture cases were of subtrochanteric fracture. One of these cases (Case 7 in Table 1) resulted in intramedullary nail conversion. However, the other two cases, which were of nondisplaced fracture, were not reoperated, and after eight weeks of non-weight-bearing, had fully recovered to normal physical activity. One case of SSI had superficial infection and was treated only by wound irrigation, which resulted in full recovery of normal physical activity. 
Several studies have attempted to identify determinants of the increased complication rate after hip fracture in the elderly; however, most evaluated populations were heterogeneous with respect to prefracture health status, living situation, and mental health [11]. Other prefracture factors frequently have acted as uncontrolled confounding variables. In addition, some complications may have been caused by technical failures.

\section{Conclusions}

The complications arising from the use of the Twins device described in this study were equivalent to the complications reported previously regarding the fixation of femoral neck fractures for elderly patients. Further studies with larger patient numbers and longer follow-up are required to determine the roles of various devices in the treatment of both nondisplaced and displaced femoral neck fractures.

\section{Abbreviations}

NSA

neck-shaft angle

SSI

surgical site infection

LSC

late segmental collapse

\section{Declarations}

- Ethics approval and consent to participate

This study was conducted with the approval of the Institutional Review Board of our institution, and all participants provided written informed consent.

- Consent for publication: Not applicable

- Availability of data and materials: Not applicable

- Competing interests: none

- Funding: none

- Authors' contributions

$\mathrm{TM}, \mathrm{MI}, \mathrm{KM}, \mathrm{KI}, \mathrm{TS}, \mathrm{TK}$ and $\mathrm{GS}$ analyzed and interpreted the patient data regarding the Xp variables. $\mathrm{HM}$, HK and YW were a major contributor in writing the manuscript. All authors read and approved the final manuscript.

- Acknowledgements: none 


\section{References}

1. Rosell PA, Parker MJ. Functional outcome after hip fracture: a 1-year prospective outcome study of 275 patients. Injury. 2003;34:529-32.

2. Merchant RA, Lui KL, Ismail NH, Wong HP, Sitoh YY. The relationship between postoperative complications and outcomes after hip fracture surgery. Ann Acad Med Singapore. 2005;34:163-8.

3. Blair B, Koval KJ, Kummer F, Zuckerman JD. Basicervical fractures of the proximal femur. A biomechanical study of 3 internal fixation techniques. Clin Orthop Rel Res. 1994:256-63.

4. Aharonoff GB, Koval KJ, Skovron ML, Zuckerman JD. Hip fractures in the elderly: predictors of one year mortality. J Orthop Trauma. 1997;11:162-5.

5. Parker MJ. The management of intracapsular fractures of the proximal femur. J Bone Joint Surg. 2000;82:937-41.

6. Conn KS, Parker MJ. Undisplaced intracapsular hip fractures: results of internal fixation in 375 patients. Clin Orthop Relat Res. 2004;421:249-54.

7. Kawasaki M, Hasegawa Y, Sakano S, Sugiyama H, Tajima T, Iwasada S, Iwata H. Prediction of osteonecrosis by magnetic resonance imaging after femoral neck fractures. Clin Orthop Relat Res. 2001;385:157-64.

8. Yamamoto T, Kobayashi Y, Nonomiya H. Undisplaced femoral neck fractures need a closed reduction before internal fixation. Eur J Orthop Surg Traumatol. 2019;29:73-8.

9. Damany DS, Parker MJ, Chojnowski A. Complications after intracapsular hip fractures in young adults: a meta-analysis of 18 published studies involving 564 fractures. Injury. 2005;36:131-41.

10. Slobogean GP, Stockton DJ, Zeng BF, Wang D, Ma B, Pollak AN. Femoral neck shortening in adult patients under the age of 55 years is associated with worse functional outcomes: Analysis of the prospective multi-center study of hip fracture outcomes in China (SHOC). Injury. 2017;48:1837-42.

11. Jacobsen SJ, Goldberg J, Miles TP, Brody JA, Stiers W, Rimm AA. Race and sex differences in mortality following fracture of the hip. Am J Pub Health. 1992;82:1147-50.

\section{Tables}

Table 1 Demographic preoperative Xp variables 


\begin{tabular}{|c|c|}
\hline Preoperative Xp variables & \\
\hline Singh's index, n (\%) & \\
\hline 1 & $1(3)$ \\
\hline 2 & $10(26)$ \\
\hline 3 & $15(38)$ \\
\hline 4 & $10(26)$ \\
\hline 5 & $3(8)$ \\
\hline 6 & none \\
\hline Garden classification, n (\%) & \\
\hline Stage $\square$ & $16(41)$ \\
\hline Stage $\square$ & $21(54)$ \\
\hline Stage $\square$ & $2(5)$ \\
\hline Stage $\square$ & none \\
\hline Mean Pauwels angle \pm SD & $40.7^{\circ} \pm 11.1^{\circ}$ \\
\hline Pauwels classification, $\mathrm{n}(\%)$ & \\
\hline Type $\square$ & $7(18)$ \\
\hline Type $\square$ & $23(59)$ \\
\hline Type $\square$ & $9(23)$ \\
\hline
\end{tabular}

Table 2 Details of reoperated cases 


\begin{tabular}{|c|c|c|c|c|c|c|c|c|}
\hline ३) & main cause & Singh's index & $\begin{array}{l}\text { Garden } \\
\text { grade }\end{array}$ & $\begin{array}{l}\text { Pauwels } \\
\text { angle } \\
\text { (degree) }\end{array}$ & $\begin{array}{l}\text { Loss of } \\
\text { reduction }\end{array}$ & $\begin{array}{l}\text { Aseptic } \\
\text { necrosis }\end{array}$ & LSC & Reoperation \\
\hline le & nonunion & 2 & प & 66 & + & + & - & $\begin{array}{l}\text { Total hip } \\
\text { arthroplasty }\end{array}$ \\
\hline & nonunion & 2 & $\square$ & 28 & + & - & - & $\begin{array}{l}\text { Bipolar head } \\
\text { replacement }\end{array}$ \\
\hline le & nonunion & 2 & 口 & 37 & - & + & + & $\begin{array}{l}\text { Bipolar } \\
\text { replacement }\end{array}$ \\
\hline le & nonunion & 3 & 口 & 30 & - & + & + & $\begin{array}{l}\text { Bipolar } \\
\text { replacement }\end{array}$ \\
\hline le & nonunion & 3 & 口 & 31 & + & - & - & $\begin{array}{l}\text { Bipolar head } \\
\text { replacement }\end{array}$ \\
\hline le & nonunion & 5 & प & 54 & - & + & + & $\begin{array}{l}\text { Bipolar head } \\
\text { replacement }\end{array}$ \\
\hline le & $\begin{array}{l}\text { subtrochanteric } \\
\text { fracture }\end{array}$ & 4 & प & 27 & - & - & - & Intramedullary nail \\
\hline le & SSI & 5 & प & 38 & - & - & - & $\begin{array}{l}\text { wound irrigation and } \\
\text { closure }\end{array}$ \\
\hline
\end{tabular}

Figures 


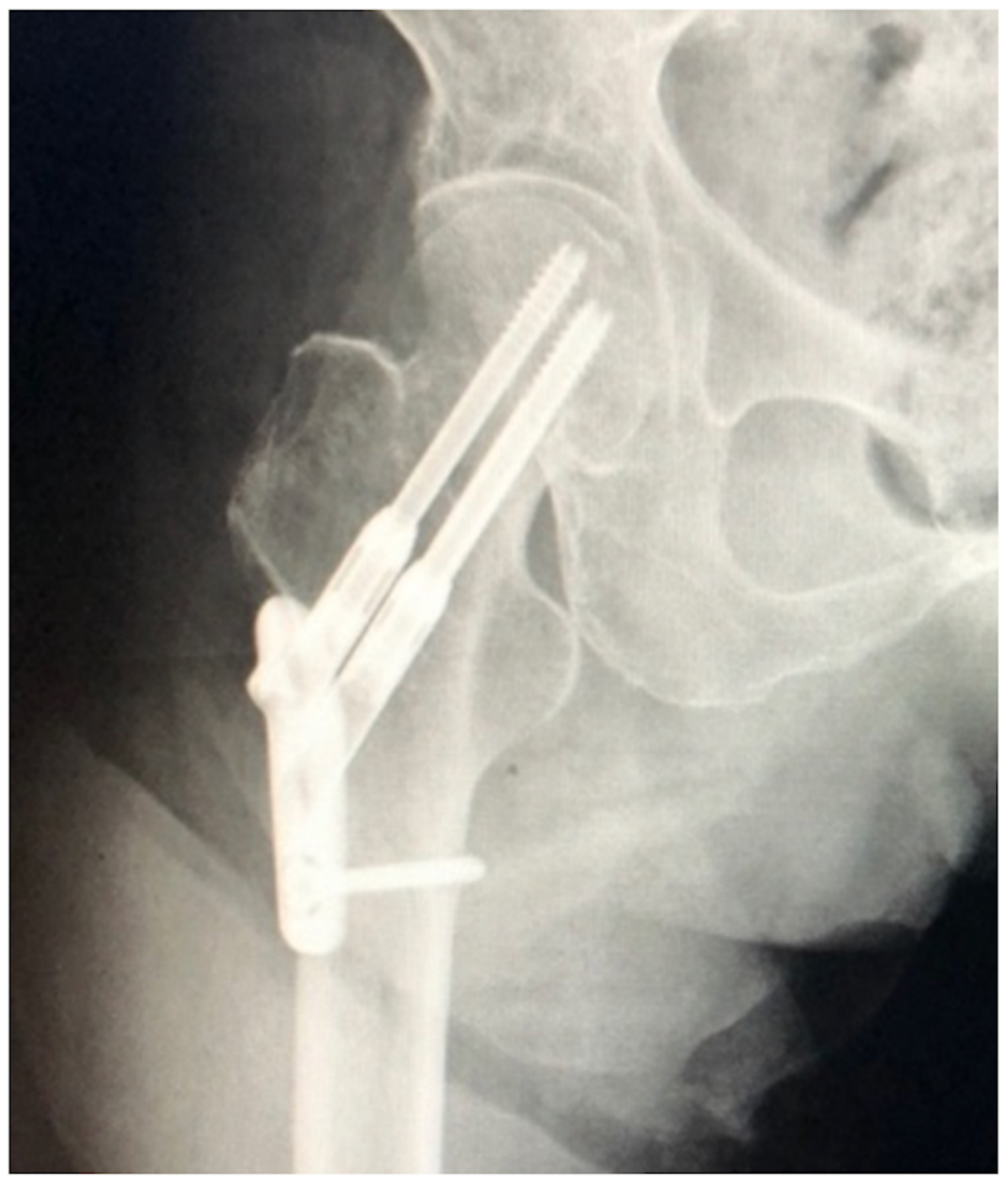

\section{Figure 1}

Femoral neck fracture fixation using Twins device. The Twins device is designed to combine twin 7-mm cancellous lag screws that telescope within a barrel, which locks to a side plate, and sliding hip screw fixation for stabilization of femoral neck fractures. The twin lag screws are oriented in an inverted parallel configuration at a 130- or 135-degree angle to the side plate. Each of the screws has $19 \mathrm{~mm}$ of available slide within the barrel when fully extended. 